Eleven consecutive patients with progressive chronic inflammatory demyelinating polyradiculoneuropathy (CIDP) underwent plasma exchange. Eight patients were previously unresponsive to prednisone, two were started on prednisone with plasma exchange, and one did not receive corticosteroids. Electrodiagnostic studies revealed evidence of an acquired demyelinating polyradiculoneuropathy with varying degrees of axonal degeneration. Neurologic impairment was monitored using conventional functional status index. Five patients demonstrated substantial clinical improvement, beginning 2 days to 3 weeks after initiating plasma exchange. Two additional patients improved following a second course of plasma exchange, and four patients demonstrated minimal or no change. Comparison of responding and nonresponding patients showed no differences related to the presence or absence of antecedent illness, duration of disease, duration of maximum weakness, or severity of impairment prior to plasma exchange. Responders had significantly prolonged F-response and motor distal latencies compared to nonresponders. Results in this unselected, consecutive patient trial suggest a temporal relationship between plasma exchange and clinical improvement in some patients with progressive CIDP.

MUSCLE \& NERVE 8:321-327 1985

\title{
PLASMA EXCHANGE IN CHRONIC INFLAMMATORY DEMYELINATING POLYRADICULONEUROPATHY
}

\author{
PETER D. DONOFRIO, MD, RUP TANDAN, MD, \\ and JAMES $W$. ALBERS, MD, PhD
}

\begin{abstract}
Chronic inflammatory demyelinating polyradiculoneuropathy (CIDP) is a diffuse, multifocal, idiopathic, peripheral nervous system disorder of presumed immunologic etiology. It is manifested as a slowly progressive or monophasic illness with stabilization in the majority of patients, but a relapsing and remitting course is seen in approximately one-third of patients. ${ }^{7}$ Frequently, CIDP follows a nonspecific infection, immunization, or exposure to immunogenic material. ${ }^{7}$ Weakness in-
\end{abstract}

From the Department of Neurology, University of Michigan, Ann Arbor MI

Presented in part at the 30th Annual Meeting of the American Association of Electromyography and Electrodiagnosis. Toronto, Canada, September 29-October 1, 1983

Dr. Tandan's present address is the University of Vermont. Department of Neurology. University Health Center, One South Prospect Street, Burlington, VT 05405

Address reprint requests to Dr. Donotrio at the Department of Neurology, University of Michigan Medical Center, 84954 CFOB. Box M056. Ann Arbor, M| 48109-0010

Received for publication June 1, 1984; revised manuscript accepted for publication August 28. 1984

0148-639x/0804/0321\$04.00/0

₹ 1985 John Wiley \& Sons. Inc. volves cranial, truncal, and proximal and distal musculature; large fiber sensory nodalities tend to be more involved than those mediated by small fibers. There is pathologic evidence of mononuclear cell infiltration, segmental demyelination, and hypertrophic changes, which are most commonly observed in spinal roots, spinal ganglia, and proximal nerve trunks. ${ }^{7}$ Electrodiagnostic testing reveals many of the following findings: severe motor and sensory conduction velocity slowing, conduction block, and dispersion of motor evoked responses on proximal stimulation; prolonged distal and F-response latencies; and abnormal sensory evoked response amplitudes. ${ }^{6.7}$

Numerous treatment modalities have been reported, including corticosteroids ${ }^{6-9.20,2: 3}$ atathioprine, ${ }^{4,6.14,18,26}$ cyclophosphamide, ${ }^{\text {ti,20 }}$ nitrogen mustard, ${ }^{20}$ poly-ICLC, ${ }^{6}$ and plasma exchange. $6.11,12,16,17,21,24,25$ All have led (t) variable results. Based on our initial experience with a patient with relapsing CIDP who demonstrated dramatic improvements following interval plasma exchange, we began a consecutive, nonrandomized trial of plasma exchange in patients with progressive CIDP. This article summarizes our results in 11 unselected patients. 


\section{mA IEMIALS AND METHODS}

Diagnostic criteria for CIDP were similar to those described by Dyck et al., ${ }^{7}$ including (1) absence of toxic exposure or associated disease commonly known to result in a neuropathy or polyradiculoneuropathy; (2) frequent history of a preceding infection or biologic material exposure; (3) steadily progressive deterioration; (4) relatively symmetric limb involvement; (5) predominance of large fiber sensory involvement; (6) cerebrospinal fluid cytoalbumin dissociation; and (7) unequivocal electrodiagnostic evidence of multifocal conduction slowing, partial or complete conduction block, and variable amounts of superimposed axonal involvement obtained using the techniques described below. None of the patients was subsequently discovered to have other causes of adult onset demyelinating polyneuropathy or polyradiculoneuropathy, i.e., diabetes, osteosclerotic myeloma, or benign monoclonal gammopathy. All patients had a chronic progressive relentless neurologic impairment for at least 3 months (range 3-22 months) prior to inclusion, and most were either corticosteroid unresponsive or intolerant. (Two patients received prednisone simultaneously with plasma exchange.) Only one patient subsequently developed a clinical course more appropriately categorized as recurrent.

A disease duration of 3 months was used to be reasonably assured that patients with acute inflammatory demyelinating polyradiculoneuropathy (AIDP) would be excluded. Most patients were referred from other institutions, where high-dose corticosteroids had been initiated for a variable period of time prior to referral. During the study period, we excluded the following patients with other forms of adult-onset demyelinating polyradiculoneuropathy (number of patients in parentheses): relapsing (IIDP and steroid-responsive CIDP (16), nonmalignant monoclonal gammopathy (7), and osteosclerotic myeloma (1).

Motor and sensory conduction studies were performed using standard techniques of supramaximal percutaneous stimulation and surface electrode recording. Compound muscle action potential (CMAP) and sensory nerve action potential (SNAP) amplitudes were measured from baseline to negative peak. Conduction velocity was measured in the forearm or leg segment, and distal latency was converted as terminal conduction velocity. Conduction study results for individual nerves were expressed a percentage of the normal mean for each nerve, then averaged and reported as a percentage of normal for motor and sensory studies.

Motor temporal dispersion was estimated as the ratio of the proximal to the distal CMAP anplitude. F-response latencies were recorded as the minimal latency in a series of $10 \mathrm{~F}$-responses using distal (wrist or ankle) antidromic motor nerve stimulation. Both temporal dispersion and $\mathrm{F}$ response latencies were averaged for individual patients. 'Temporal dispersion was reported as an absolute percentage; F-response latency was reported as a percentage of the upper limit of normal. Median (motor and sensory), ulnar (motor), peroneal (motor), tibial and sural nerves were evaluated.

Criteria for demyelination required demonstration of at least one of the following in two or more nerves (exceptions noted):

1. Conduction velocity less than $95 \%$ of lower limit of normal if amplitude exceeded $50 \%$ of lower limit of normal, less than $85 \%$ if amplitude less than $50 \%$ of lower limit of normal. Excluded were isolated ulnar or peroneal nerve abnormalities at the elbow or knee, respectively.

2. Distal latency exceeding $110 \%$ of upper limit of normal if amplitude normal, exeeding $120 \%$ of upper limit of normal if amplitude less than lower limit of normal. Excluded were isolated median nerve abnormalities at the wrist.

3. Evidence of excessive motor nerve temporal dispersion, defined as a proximal to distal CMAP amplitude ratio less than $70 \%$. Excluded were isolated ulnar or peroneal lesions, as above, as well as any studies with evidence of anomalous innervation (e.g., median to ulnar nerve crossover).

4. F-response latency exceeding $120 \%$ of upper limit of normal with combined exclusions from above.

All needle electromyography recordings were performed using standard concentric needle electrodes. Spontar:uus activity (fibrillation potentials or positive :aves) were recorded in a given muscle as $0=$ none, $1+=$ persistent single trains in at least two areas away from the endplate, $2+=$ moderate numbers in three or more areas, $3+=$ many in all areas, and $4+=$ filling the baseline in all areas. Motor unit action potential (MUAP) recruitment was graded subjectively using $0=$ none, $1+=$ severe decrease, $2+=$ moderate decrease, $3+=$ mild decrease, and $4+=$ normal. Spontaneous activity and MUAP recruitment was re- 
Table 1. Clinical grading of neurologic impairment or improvement.

\begin{tabular}{ll}
\hline & Functional status index $(\mathrm{FSI})$ \\
\hline 0-Normal & 8-Crutches \\
1-Walk alone (minimal impairment) & 9-Parallel bars \\
2-Walk alone (mild weakness) & 10-Assistance \\
3-Walk alone (moderate weakness) & $11-$ Wading tank \\
4-Walk alone (moderate impairment) & $12-$ Wheelchair \\
5-Walk alone (marked impairment) & 13 - Confined to bed \\
6-Cane & 16-Respirator \\
7-Walker & 21-Dead \\
Substantial clinical improvement: change of at least 3 FSI \\
grades
\end{tabular}

ported as an average for proximal and distal muscles. For all studies, skin temperatures were measured and maintained above $32^{\circ} \mathrm{C}$ using either warm compresses or an infrared heater.

Plasma exchange was executed using either an IBM 2997 Blood Cell Separator (International Business Machine Biomedical Systems, Cranbury, NJ) or a Haemonetics Model 30 Blood Cell Separator (Haemonetics Corp., Braintree, MA). Each patient received five separate exchanges of one plasma volume per exchange over a 10-14-day course. As a second course of plasma exchange, patient 6 received one plasma volume exchange per month for five consecutive months. Replacement fluid consisted of $5 \%$ normal serum albumin and, when necessary for hypofibrinogenemia, $2 \mathrm{U}$ of single donor plasma.

Neurologic impairment was numerically assessed using a conventional functional status index (FSI; ' $\Gamma$ able 1).

Clinical Characteristics. There were four males and seven females, having an average age of 50 years (range 18-73). Major clinical manifestations are summarized in 'Table 2. Approximately half of the patients described an identifiable antecedent event (either surgery or respiratory tract infection) predating their neurologic illness by days to weeks. Initial symptoms were sensory in five patients, motor in five, and combined sensory and motor in the remaining patient. Impaired gait was the initial motor complaint in all patients. Sensory deficits involved presumed small and large fiber modalities, with abnormalities of the latter predominating. Autonomic symptoms and signs, sensory tremor, and respiratory impairment were relatively infrequent. Cranial nerve abnormalities included facial diplegia (three patients), equivocal orbicularis oculi weakness (eight patients), and pharyngeal weakness (one patient).

Analyses of blood and urine were unremarkable; no patients were subsequently found to have diabetes, vasculitis, osteosclerotic myeloma, or a benign monoclonal gammopathy. Cerebral spinal fluid (CSF) protein was elevated in all patients.

Eight patients had been treated with high-dose corticosteroids prior to plasma exchange and were continued on this dosage during and for 1 month following plasma exchange, whereafter a tapering dosage schedule similar to that suggested by Dalakas and Engel ${ }^{6}$ was initiated. No other immunosuppressants were prescribed. Two patients were started on high-dose corticosteroids $(60)$ and $80 \mathrm{mg}$ prednisone) within days of plasma exchange; in a third patient, corticosteroid treatment was judged to be contraindicated (prior gastrointestinal bleeding).

\section{RESULTS}

Seven of the 11 patients demonstrated clinical improvement following plasma exchange (Table 3),

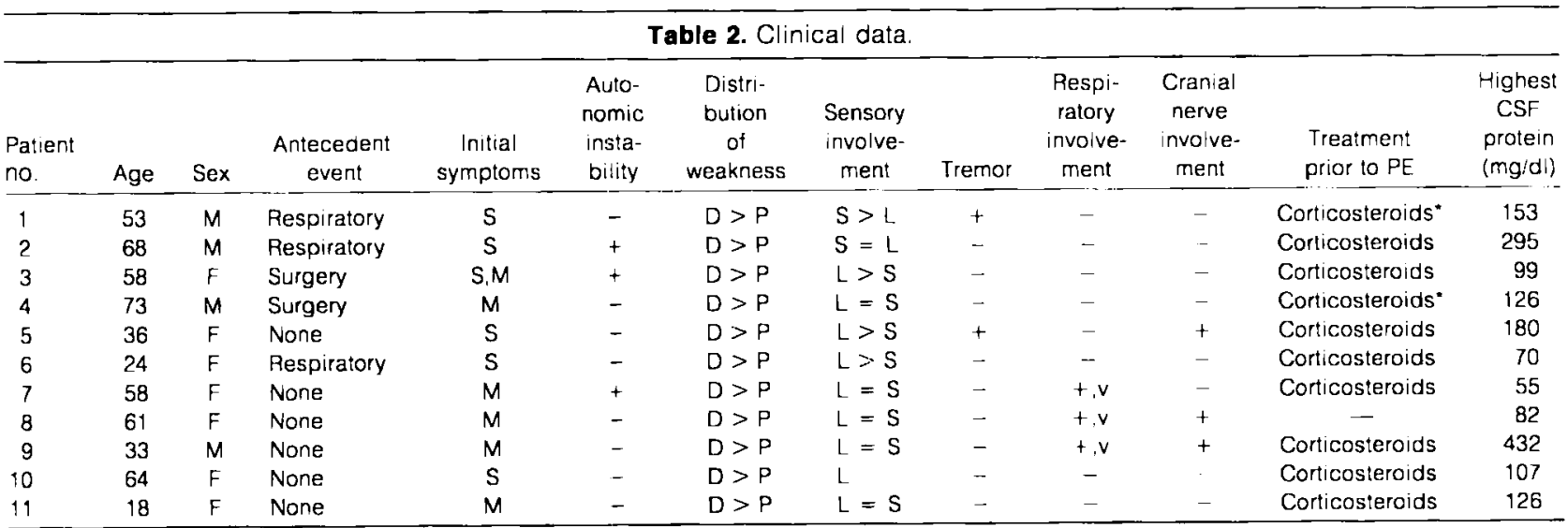

$S$, sensory, $M$, motor: $D$, distal; $P$, proximal; + , present. - absent: $L$. large hber, $S$, small fiber; $V$, ventivaror dependent; $P E$. plasma exchange - Simultaneous with onset of plasma exchange 


\begin{tabular}{|c|c|c|c|c|c|c|c|c|c|c|c|c|}
\hline \multirow{2}{*}{$\begin{array}{l}\text { Patient } \\
\text { no. }\end{array}$} & \multirow{2}{*}{$\begin{array}{l}\text { Onset } \\
\text { to PE } \\
\text { (mo) }\end{array}$} & \multirow{2}{*}{$\begin{array}{l}\text { Onset } \\
\text { to peak } \\
(\mathrm{mo})\end{array}$} & \multirow{2}{*}{$\begin{array}{l}\text { Duration } \\
\text { plateau } \\
\text { (mo) }\end{array}$} & \multirow{2}{*}{$\begin{array}{c}\text { Time following } \\
\text { PE to } \\
\text { improvement (days) }\end{array}$} & \multirow{2}{*}{$\begin{array}{c}\text { FSI } \\
\text { pre-PE }\end{array}$} & \multicolumn{5}{|c|}{$\begin{array}{l}\text { Scores (FSI) } \\
\text { following PE }\end{array}$} & \multirow{2}{*}{$\begin{array}{l}\text { Change in } \\
\text { FSI at } \\
3 \text { mo }\end{array}$} & \multirow{2}{*}{$\begin{array}{l}\text { Substantial } \\
\text { improvement } \\
>3 \mathrm{FSI}\end{array}$} \\
\hline & & & & & & 1 & 2 & 3 & 6 & 12 & & \\
\hline 1 & 8 & 8 & 0 & 10 & 13 & 6 & 6 & 6 & 3 & 1 & +7 & Yes \\
\hline 2 & 7 & 5 & 2 & 20 & 12 & 10 & 9 & 7 & 6 & $\bullet$ & +5 & Yes \\
\hline 3 & 5 & 3 & 2 & 14 & 12 & 9 & 8 & 6 & 5 & 5 & +6 & Yes \\
\hline 4 & 7 & 7 & 0 & $20-25$ & 13 & 12 & 12 & 7 & 5 & 4 & +6 & Yes \\
\hline \multirow[t]{2}{*}{5} & 7.5 & 4 & 3.5 & - & 8 & 8 & & & & & - & \\
\hline & & & & $1-2$ days & 9 & 5 & 4 & 4 & 3 & 1 & +5 & Yes (second PE) \\
\hline \multirow[t]{2}{*}{6} & 10 & 10 & 0 & - & 7 & 8 & 8 & 8 & - & - & -1 & \\
\hline & & & & $\dagger$ & 8 & 8 & - & 5 & 5 & 5 & +3 & Yes (second PE) \\
\hline 7 & 11 & 0.1 & 11 & - & 16 & 13 & 13. & 13 & 21 & 21 & +3 & No \\
\hline 8 & 8 & 5 & 3 & - & 16 & 16 & 16 & 16 & - & 21 & 0 & No \\
\hline 9 & 4 & 4 & 0 & - & 13 & 13 & 13 & 16 & 21 & - & -3 & No \\
\hline 10 & 22 & 22 & 0 & 2 & 2 & 2 & 2 & 1 & 1 & 1 & +1 & No \\
\hline 11 & 3 & 3 & Few days & 2 & 5 & $*$ & 2 & 1 & 0 & $*$ & +4 & Yes \\
\hline
\end{tabular}

*No follow-up.

†During second course of PE (1 exchange/mo for $5 \mathrm{mo}$ ).

using an arbitrary definition of improvement as an increase of three or more points on the FSI scale by the third month following plasma exchange. Three months was chosen because of previous experience with plasma exchange in another patient with the relapsing form of CIDP (not included in this study). In this patient, objective clinical improvement was noted not only 1-2 weeks post plasma exchange, but also steadily for several months. Thus, three months represented a sufficient time frame for observation of initial, prolonged, and potentially delayed improvement. Progressive improvement occurred in the setting of a steroid taper in all responding patients. Patient 7 was not classified as a responder to plasma exchange, because her change in FSI scale merely reflected weaning from mechanical ventilation, without an improved forced vital capacity or improved extremity function. Initial improvement occurred on an average of 12 days following initiation of plasma exchange. Two patients noted subjective improvement within 2 days of treatment. Both patients who were simultaneously started on corticosteroids and plasma exchange improved; the degree of improvement did not differ from that of patients who were previously steroid unresponsive or intolerant. In addition, two other patients who were unresponsive to an initial trial of plasma exchange improved after or during a second course of plasma exchange. No other immunosuppressant medications were administered with plasma exchange. Response to plasma exchange was not related to any of the following: duration of disease prior to plasma exchange, time from onset to maximum neurologic deficit, duration of maximum weakness plateau, or FSI score prior to plasma exchange.

Analysis, as a separate group, of the eight patients who were previously refractory to high-dose corticosteroids disclosed response to plasma exchange in five patients. Improvement (average 3 FSI grades) was noted 1 month subsequent to plasma exchange in three patients (patient 5 following the second course of exchange) and in all five patients at 3 months (average 4.6 FSI grades) and 6 months (average 5.4 FSI grades). Two of the three patients not responding to plasma exchange died within 6 months of treatment secondary to severe unrelated medical problems. At 3- and 6month follow-ups, patient 10 showed a 1 grade FSI improvement while undergoing corticosteroid tapering.

Plasma exchange was free of serious complication in all 11 patients. On one occasion, patient 3 developed a transient urticarial reaction to single donor plasma adminstered for hypofibrinogenemia.

The electrodiagnostic findings for patients responding and those not responding to plasma exchange are shown in Table 4. Taken together, none of the measures was significantly $(P<0.05)$ different from results obtained previously from 18 patients with a clinical diagnosis of CIDP who were not treated with plasma exchange. Responding patients did demonstrate significantly reduced conduction, as measured by F-response latency and terminal conduction velocity, when compared with nonresponding patients. Conduction velocity (as 


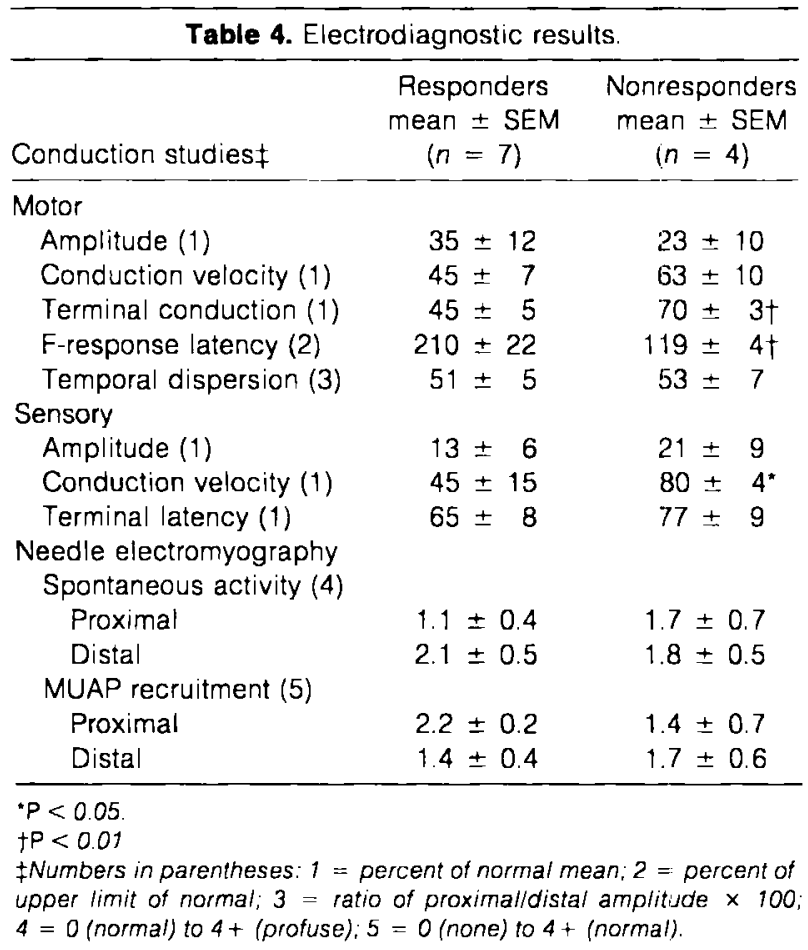

measured in the forearm and leg, respectively) was correspondingly reduced in responding patients, but did not reach statistical significance.

Serial pre- and post-plasma exchange electrodiagnostic studies were performed on all but one patient (patient 9). Of the seven patients clinically responding to plasma exchange, six demonstrated improved conduction study results for multiple nerve assessment in at least two (average 5.3) of the following parameters: evoked amplitude, terminal conduction, conduction velocity, and Fresponse latency. The sole patient (patient 4) with deterioration in electrodiagnostic results despite clinical improvement following plasma exchange nevertheless showed an impressive clinical change (FSI improvement of 6 grades). 'The most common parameters reflecting improvement were motor and sensory evoked amplitudes and terminal conduction.

\section{DISCUSSION}

CIDP has been treated with several therapeutic agents since its original description. ${ }^{2,3}$ Prineas and McLeod $^{20}$ described improvement in 13 of $17 \mathrm{pa}^{-}$ tients with relapsing CIDP treated with prednisone or ACTH. In the largest series to date of patients with CIDP, Dyck et al. ${ }^{7}$ reported improvement following corticosteroid use in $39 \%$ of patients. Using objective clinical and electrodiagnostic monitoring, they demonstrated a small, but significant improvement over no treatment in a 3-month trial of prednisone in 28 patients with CIDP. ${ }^{8}$ Other reports have documented similar results in smaller trials. $^{6,9,23}$

Several case reports and small series describe reversal of symptoms and signs in CIDP with azathioprine. ${ }^{1,6,18,26}$ In several instances, azathioprine produced improvement in patients who were previously unresponsive to steroids or allowed replacement of steroids with azathioprine. ${ }^{\text {b. } 18}$ Less commonly prescribed, but occasionally effective, medications include cyclophosphamide, ${ }^{6,20}$ nitrogen mustard ${ }^{20}$ and poly-ICI.C. ${ }^{6}$

Several case reports describe improvement in CIDP following plasma exchange. ${ }^{11,16,17,21}$ Included are patients treated with plasma exchange only, or patients treated in combination with corticosteroids or cyclophosphamide. In the report of Levy et al., ${ }^{16}$ the clinical disease course had been refractory to previous corticosteroid administration. Only one of three CIDP patients reported by Dalakas and Engel ${ }^{6}$ improved following plasma exchange, and this improvement was characterized as minimal. In addition, only one of two patients described by Gross et al. ${ }^{12}$ improved subsequent to plasma exchange. In the largest series to date of plasma exchange in CIDP, marked clinical improvement (two patients) and partial improvement (three patients) occurred in five of seven patients undergoing plasma exchange. ${ }^{25}$ The use of supplemental corticosteroids or other immunosuppressants was not described.

Plasma exchange is of unproven benefit in CIDP. Nonetheless, our experience with 11 unselected, consecutive patients suggests that plasma exchange may result in rapid improvement, even in patients with a chronic progressive history who were previously unresponsive or intolerant to corticosteroids. None of our patients had been previously treated or had plasma exchange supplemented with other immunosuppressants.

A surprising result from this study was the observation that plasma exchange may result in continued improvement beyond the first weeks following exchange. All seven patients responding to plasma exchange showed progressive improvement beyond the first month subsequent to exchange, and several continued to gain strength beyond the 6-month follow-up. This improvement evolved in the setting of a tapering of the dose of corticosteroids. Because the mechanism of the benefit of plasma exchange is still unknown in demyelinating neuropathies, a satisfactory explana- 
tion for the prolonged effect of plasma exchange eludes us. Possibly, repair and remyelination of peripheral nerve transpires slowly over months, or "antibody" removal by plasma exchange is not its sole mechanism of action.

Also unexplained are the electrodiagnostic findings that patients responding to plasma exchange statistically had longer F-response latencies and slower motor terminal conduction. This result suggests a fascinating hypothesis that plasma exchange may be most beneficial in reversing extensive proximal and distal demyelination. Whether or not potential responders to plasma exchange in CIDP might be predicted on the basis of nerve conduction results awaits more controlled studies on larger patient populations.

At present, removal of a presumed antibody directed against peripheral nerve myelin is the most intuitively satisfying explanation for improvement following plasma exchange in CIDP. Pathologic studies in CIDP have revealed features similar to those observed in AIDP and in animals with experimental allergic neuritis (EAN), namely, scattered areas of circumscribed myelin lost in the presence of lymphocytes and macrophages, prominent onion bulb formation, and axonal loss. ${ }^{19}$ These findings strongly suggest an immunologic process, but it is unclear whether or not that process is primarily humoral, cell-mediated, or a combination of both.

Dalakas and Engel, ${ }^{6}$ on examining frozen sections of sural nerve biopsies of nine patients with CIDP, found linear depositions of IgM in six patients and $\operatorname{IgG}$ in three patients on what appeared to be the exterior portion of the Schwann cell plasmalemma. They postulated that the major antigen in CIDP may be a superficial plasmalemmal carbohydrate group that elicits a persistent $\operatorname{IgM}$ response, with subsequent immunoglobulin binding to the Schwann cell surface. Demyelination in rat sciatic nerve has been demonstrated following passive transfer of fresh serum from patients with AIDP, yet whether or not serum from patients with CIDP also produces demylination when injected into nerve is unknown. ${ }^{10,13}$ Thus far, antimyelin antibodies have not been reported in CIDP, and Leibowitz and Hughes ${ }^{15}$ could not demonstrate antibodies to $P_{2}$ by radioimmunoassay or to galactocerebroside by a complement-fixing technique. Whether or not an antibody is the initiator of myelin damage in CIDP or AIDP is uncertain. Yonezawa et al., ${ }^{27}$ in an EAN model, detected a serum factor which caused macrophages to adhere to and demyelinate normal myelin sheaths in vitro.

Cell-mediated immunity studies in patients with CIDP have also demonstrated a heightened response. Sheremata et al. ${ }^{22}$ reported enhanced macrophage migration inhibition factor release to a homogenate of human sciatic nerve in the lymphocytes of one patient with CIDP. In addition, lymphocyte transformation was magnified on exposure to CNS MBP and a peripheral nerve MBP (myelin basic protein) in four patients with CIDP. ${ }^{1}$

Clarification of the efficacy of plasma exchange in progressive CIDP awaits further prospective randomized trials. The identification of apparent electrodiagnostic differences between responding and nonresponding patients in this unselected consecutive patient trial suggests that these measures must be controlled or closely evaluated in future randomized studies.

\section{REFERENGE:}

1. Abranıky O, Webb C, Teitelbaum D, Arnon R: Cellmediated immunity to neural antigens in idiopathic polyneuritis and myeloradiculitis. Neurology (Minneap) 25:1154. 1975.

2. Austin JH: Observations on the syndrome of hypertrophic neuritis (the hypertrophic interstitial radiculo-neuropathies). Medicine (Baltimore) 35:187-237, 1956.

3. Austin JH: Recurrent polyneuropathies and their corticosteroid treatment - With five-year observations of a placebo-controlled case treated with corticotrophin, cortisone and prednisone. Brain 81:157-194, 1958

4. Cendrowski W: Treatment of polyneuropathy with azathioprine and adrenal steroids. Acta Med Pol 18:147-156. 1977.

5. Cook SD, Dowling PC: The role of autoantibody and immune complexes in the pathogenesis of Guillain-Barte syndrome. Ann Neurol 9(suppl):70-79. 1981

6. Dalakas MC, Engel WK: Chronic relapsing (dysimmune) polyneuropathy: Pathogenesis and treatment. Ann Neurol 9 (suppl): $134-135,1981$.

7. Dyck PJ, Lais AC, Ohta M, Bastron JA, Okazaki H, Groover RV: Chronic intlammatory polyradiculoneuropathy. Mayo Clin Proc 50:621-637, 1975.

8. Dyck PJ, O'Brien PC, Oviatt KF, Dinapoli RP, Daube JR, Bartleson JD, Mokri B, Swift T, Low PA, Windebank AJ: Prednisone improves chronic inflammatory denyelinating polyradiculoneuropathy more than no treatment. Ann $\mathrm{Neu}$ rol $11: 136-141,1982$.

9. Dyck PJ, Swanson CJ, Low PA, Bartleson JD, Lambert EH: Prednisone-responsive hereditary motor and sensory neuropathy. Mayo Clin Pror 57:239-246, 1982.

10. Feasby TE, Hahn AF, Gilbert JJ: Passive transfer of demyelinating activity in Guillain-Barre polyneuropathy. Neurology (Minneap) 30:363, 1980.

11. Fowler H, Vulpe M, Marks (;, Egole C;, Dau PC: Recovery from chronic progressive polyneuropathy after treatment 
with plasma exchange and cyclophosphamide. Lancet 2:1193, 1979.

12. Gross MLP, Legg NJ, Lockwood MC, Pallis C: The treatment of inflammatory polyneuropathy by plasma exchange. J Neurol Neurosurg Psychiatry 45:675-6i79, 1982.

13. Harrison BM, Hansen LA, Pollard JD, McLeod JG: Demyelination induced by serum from patients with GuillainBarre syndrome. Ann Neurol 15:163-170, 1984.

14. Heathfield K, Dallos V: Treatment of polyneuropathy with azathioprine. Lancel 2:1030-1031, 1970

15. Leibowitz S, Hughes RAC: Experimental allergic neuritis, human acute polyradiculoneuritis and related disorders, in Immunology of the Neruous System, chapter 5. London, Edward Arnold, 1983, pp 122-123.

16. Levy RL, Newkirk R, Ochna J: Treating chronic relapsing Guillain-Barre syndrome by plasma exchange. Lancet $2: 259-260,1979$.

17. Mark B, Hurwitz BJ, Olanow C'W, Fay JW: Plasmapheresis in idiopathic inflammatory polyradiculoncuropathy. Neurology (Minneap) 30:361, 1980 .

18. Pentland B, Adams GGW, Mawdsley C: Chronic idiopathic polyneuropathy treated with azathioprine. I Neurol Neurosurg Psychiatry 45:866-869, 1982.

19. Prineas JW: Pathology of the Guillain-Barre syndrome. Ann Neurol 9(suppl):6-19, 1981.

20. Prineas JW, McLeod JG: Chronic relapsing polyneuritis. $J$ Neurol Sri 27:427-458, 1976.
2I. Server AC, Stein SA, Braine H, Tandon DS, McKhanI GM: Experience with plasma exchange and cyclophosphamide in the treatment of chronic relapsing inflammatory polyradiculoneuropathy. Neurology (Minneap) 30:362, 1980.

22. Sheremata WA, Colby S, Lusky G, Cosyrove JBR: Cellular hypersensitivity to peripheral nerve antigens in the (;BS. Neurolugy (Minneap) 25:833, 1975.

23. Thomas PK, Lascelles RG, Hallpike JF, Hewer RI.: Recurrent and chronic relapsing Guillain-Barré polyneuritis. Brain 92:589-606, 1969.

24. Toyka KV, Augspach R, Wietholter H, Besinger UA, Haneveld F, Liebert UG, Heininger K, Schwendemann $(\dot{B}$, Reiners K, Grabensee B: Plasma exchange in chronic inflammatory polyneuropathy: Evidence suggestive of a pathogenic humoral factor. Muscle Nerve 5:479-484, 1982.

25. Vedeler CA, Nyland II, Fagius J, Oterman PO, Matre R, Aarli JA, Janzen RWC, Jacobsen H, Skre H: The clinical effect and the effect on serum IgG $\mathrm{i}$ antibodies to peripheral nerve tissue of plasma exchange in patients with GuillainBarré syndrome. J Neurol 228:59-64, 1982.

26. Walker GL: Progressive polyradiculoneuropathy: 'Treatment with azathioprine. Aust $N Z J$ Med 9:184-187, 1979.

27. Yonezawa $T$, Ishihara $Y$, Matsuyama $\mathrm{H}$ : Studies on experimental allergic peripheral neuritis. (1) Demyelinating patterns studies in vitro. J Neuropathol Expi Neurol 27:453-463. 1968. 